diazepam IV, oxygen, and stabilize airway and respiration as needed. Check serum glucose and begin EKG monitoring. For established SE, 5-10 min, repeat benzodiazepine, give fosphenytoin IV. Consider pyridoxine $100 \mathrm{mg}$ IV push, if age $<2$ years. Obtain lab tests, including CT and neurology consult. For refractory SE, $10 \mathrm{~min}$ after fosphenytoin infusion, administer levetiracetam IV or valproate. After $5 \mathrm{~min}$ interval without control, give phenobarbital IV. Admit to PICU. Consider coma induction. Inhalation anesthetics will terminate refractory SE and induce burst suppression, but hypotension requiring vasopressors is a problem, and seizures recur when the anesthetic is withdrawn. (Abend NS, Dlugos DJ. Treatment of refractory status epilepticus: literature review and a proposed protocol. Pediatr Neurol June 2008;38:377-390). (Respond: Dr Abend, Division of Neurology, Children's Hospital of Philadelphia, 34 ${ }^{\text {th }}$ St and Civic Center Blvd, Philadelphia, PA 19104. E-mail: abend(iemail.chop.edu).

COMMENT. The treatment protocol used at the Philadelphia Children's Hospital for status epilepticus emphasizes the benefits of early intervention, consecutive medications with different mechanisms of action, and avoidance of risk of hypotension. Status epilepticus due to recognized causes (eg febrile SE, meningitis, encephalitis, etc) require specific treatment.

\title{
CLINICAL FEATURES OF PROLONGED FEBRILE SEIZURES
}

The results of a prospective, multicenter study of 119 children (age 1 month through 5 years) with prolonged febrile seizures (30 min or longer) are reported from Montefiore Medical Center, and Columbia University, New York; Children's Memorial Hospital, Chicago; and other members of the FEBSTAT study group. The median age was 1.3 years; $46 \%$ between 1-2 years. Males were 54\%. Development was normal in $86 \%$; and definitely abnormal in $8 \%$. A prior FS occurred in $24 \%$. Family history was positive for FS in $25 \%$ and for epilepsy in $9 \%$. The mean peak temperature was $103.2 \mathrm{~F}$. The cause of the fever was viral in $54 \%$, otitis media $(18 \%)$, pneumonia $(5 \%)$, and unknown $(14 \%)$. Median duration of the FS was $68 \mathrm{~min} ; 24 \%$ were $>2$ hours. A diagnosis of status epilepticus was not recognized in the ED in $21 \%$. Seizures were convulsive in $99 \%$, definitely focal in $48 \%$, generalized in $22 \%$, and partial with secondary generalization in $66 \%$. (Shinnar S, Hesdorffer DC, Nordli DR Jr, et al. Phenomenology of prolonged febrile seizures. Results of the FEBSTAT study. Neurology July 15, 2008;71:1-1 [Epub ahead of print]. (Respond: Shlomo Shinnar MD, Comprehensive Epilepsy Management Center, Montefiore Medical Center, 111 E $210^{\text {th }}$ St, Bronx, NY 10467. E-mail: sshinnar@aol.com).

COMMENT. This report represents a preliminary account of a long-term study to include 200 patients with prolonged febrile seizures, and is intended to clarify the relation of the FS to mesial temporal sclerosis and temporal lobe epilepsy. Febrile status epilepticus accounts for $25 \%$ of pediatric SE patients. The high incidence of partial seizures might be expected, given the prolonged nature of the seizures in this study. Hippocampal injury is reported especially in patients with a history of prolonged, focal FS. However, inter-rater agreement on focality reached lesser consensus than other features of FS. The high incidence of viral infection is in agreement with most recent FS studies. The results of viral assays will be of interest and important information, as a prelude to future research in viral etiology and antiviral treatment of FS. 